PROCEEDINGS OF THE

AMERICAN MATHEMATICAL SOCIETY

Volume 135, Number 9, September 2007, Pages 2751-2757

S 0002-9939(07)08775-8

Article electronically published on March 30, 2007

\title{
ON COMPLEMENTED VERSIONS OF JAMES'S DISTORTION THEOREMS
}

\author{
WILLIAM B. JOHNSON AND NARCISSE RANDRIANANTOANINA
}

(Communicated by N. Tomczak-Jaegermann)

\begin{abstract}
Examples are given to show that two natural questions asked in Dowling, Randrianantoanina, and Turett, 1999, about complemented versions of James's distortion theorems have negative answers.
\end{abstract}

\section{INTRODUCTION}

The James's distortion theorem for $\ell_{1}$ (respectively, for $c_{0}$ ) states that whenever a Banach space contains a subspace isomorphic to $\ell_{1}$ (respectively, $c_{0}$ ) then the Banach space contains subspaces that are almost isometric to $\ell_{1}$ (respectively, $c_{0}$ ). In [5], complemented versions of James's distortion theorems were considered in the following senses:

Theorem 1. Let $X$ be a Banach space whose dual unit ball is weak-sequentially compact and $\varepsilon>0$. If $X$ contains a subspace isomorphic to $c_{0}$, then there exists a subspace $Z$ of $X$ and a projection $P$ from $X$ onto $Z$ such that $Z$ is $(1+\varepsilon)$-isometric to $c_{0}$ and $\|P\| \leq 1+\varepsilon$. Moreover, if $X$ contains a subspace isometric to $c_{0}$, then there exists a subspace $Z$ of $X$ and a projection $P$ from $X$ onto $Z$ such that $Z$ is isometric to $c_{0}$ and $\|P\|=1$.

Theorem 2. Let $X$ be a Banach space which contains a complemented subspace isomorphic to $\ell_{1}$ and $\varepsilon>0$. Then there exists a subspace $Y$ of $X$ and a projection $P$ from $X$ onto $Y$ such that $Y$ is $(1+\varepsilon)$-isometric to $\ell_{1}$ and $\|P\| \leq 1+\varepsilon$.

While Theorem 2 can be viewed as the exact analogue of James's distortion theorem for complemented copies of $\ell_{1}$, Theorem 1 may be interpreted as a combination of the James's distortion theorem for $c_{0}$ and the classical Sobczyk Theorem. These led to the following natural questions (see [5, Question 1, Question 2]):

Question 1. If a Banach space $X$ contains a complemented copy of $c_{0}$ and if $\varepsilon>0$, does there exist a subspace $Z$ of $X$ and a projection $P$ from $X$ onto $Z$ such that $Z$ is $(1+\varepsilon)$-isometric to $c_{0}$ and $\|P\| \leq 1+\varepsilon$ ?

Received by the editors January 10, 2006 and, in revised form, May 1, 2006.

2000 Mathematics Subject Classification. Primary 46B20, 46B25: Secondary 46B03, 46 B45.

Key words and phrases. $\ell_{1}, c_{0}$, renorming, James's distortion theorems.

The first author's research was partially supported by NSF DMS-0503688.

The second author's research was partially supported by NSF DMS- 0456781 . 
Question 2. If a Banach space $X$ contains a complemented subspace isometric to $\ell_{1}$, does there exist a subspace $Z$ of $X$ and a projection $P$ from $X$ onto $Z$ such that $Z$ is isometric to $\ell^{1}$ and $\|P\|=1$ ?

The aim of this note is to provide examples showing that, as expected, the answers to both questions are negative.

\section{The EXAMPLeS}

2.1. The $c_{0}$-case. In this subsection, we exhibit a Banach space $X$ with the property that any complemented subspace of $X$ that is almost isometric to $c_{0}$ has a large projection constant, thus answering Question 1 negatively. The space $X$ is a renorming of $\ell_{\infty} \oplus_{\infty} c_{0}$.

We denote by $\|\cdot\|$ the usual norm on $\ell_{\infty} \oplus_{\infty} c_{0}$. In order to define the new norm on $X$, let $J: \ell_{1} \rightarrow \ell_{\infty}$ be an isometric embedding of $\ell_{1}$ into $\ell_{\infty}$ and $Q: \ell_{1} \rightarrow c_{0}$ be a quotient map. For $\delta>0$, a norm $\||\cdot|||$ on $X$ is defined by fixing its unit ball:

$$
B_{(X,\|\cdot\| \|)}:=\left\{(J f, Q f) ; f \in \ell_{1},\|f\|_{1} \leq 1\right\}+\delta B_{(X,\|\cdot\|)} .
$$

It is clear that $|\|\cdot \mid\|$ and $\|\cdot\|$ are equivalent norms on $X$ and $X$ contains a complemented subspace isomorphic to $c_{0}$.

Proposition 3. Let $\varepsilon>0$ and $Z$ be a subspace of $(X,\||\cdot|\| \mid)$ that is $(1+\varepsilon)$-isometric to $c_{0}$ and is complemented in $X$. If $P$ is a projection from $X$ onto $Z$, then

$$
\||P|\| \geq \frac{1+\delta}{4 \delta(1+\varepsilon)^{3}}
$$

Proof. Throughout, we also denote by $|\|\cdot\|| \mid$ the corresponding dual norm on $X^{*}$. Let $\left(V_{n}\right)_{n>1}$ be a basic sequence $(1+\varepsilon)$-equivalent to the unit vector basis of $c_{0}$ and whose closed linear span is $Z$. Let $P$ be a projection from $X$ onto $Z$. Then $P$ is of the form

$$
P=\sum_{n=1}^{\infty} V_{n}^{*} \otimes V_{n}
$$

where $\left(V_{n}^{*}\right)_{n \geq 1}$ is a weak ${ }^{*}$-null sequence in $X^{*}$ and the sum can be taken with respect to the strong operator topology. Observe that $X^{*}=\left(\ell_{\infty}\right)^{*} \oplus_{1} \ell_{1}$ isomorphically and thus for every $n \geq 1, V_{n}^{*}=\left(x_{n}^{*}, a_{n}^{*}\right)$ where $\left(x_{n}^{*}\right)_{n \geq 1}$ (respectively, $\left.\left(a_{n}^{*}\right)_{n \geq 1}\right)$ is a weak ${ }^{*}$-null sequence in $\left(\ell_{\infty}\right)^{*}$ (respectively, $\left.\ell_{1}\right)$. Since weak ${ }^{*}$-null sequences are weakly-null in $\left(\ell_{\infty}\right)^{*}$ (see for instance [1, Theorem 15, p.103]), we have

$$
\text { weak }-\lim _{n \rightarrow \infty} x_{n}^{*}=0 .
$$

There exists a convex block $\left(y_{n}^{*}\right)_{n \geq 1}$ of $\left(x_{n}^{*}\right)_{n \geq 1}$ with

$$
\lim _{n \rightarrow \infty}\left\|y_{n}^{*}\right\|=0 .
$$

There exists a strictly increasing sequence of integers $\left(k_{n}\right)_{n \geq 0}$ and positive scalars $\alpha_{j}^{(n)}$, where $k_{n-1}+1 \leq j \leq k_{n}, \sum_{j=k_{n-1}+1}^{k_{n}} \alpha_{j}^{(n)}=1$ for $n \geq 1$, and

$$
y_{n}^{*}=\sum_{j=k_{n-1}+1}^{k_{n}} \alpha_{j}^{(n)} x_{j}^{*} .
$$


For $n \geq 1$, consider the corresponding block sequences:

$$
\begin{aligned}
& W_{n}=\sum_{j=k_{n-1}+1}^{k_{n}} V_{j}, \\
& W_{n}^{*}=\sum_{j=k_{n-1}+1}^{k_{n}} \alpha_{j}^{(n)} V_{j}^{*} .
\end{aligned}
$$

Then $\left(W_{n}\right)_{n \geq 1}$ is equivalent to the unit vector basis of $c_{0}$. Moreover, for every $n, k \geq 1,\left\langle W_{n}^{*}, W_{k}\right\rangle=\delta_{n}^{k}, W_{n}^{*}=\left(y_{n}^{*}, b_{n}^{*}\right)$ where $b_{n}^{*}=\sum_{j=k_{n-1}+1}^{k_{n}} \alpha_{j}^{(n)} a_{j}^{*}$, and $\left\|W_{n}\right\| \| \leq 1+\varepsilon$. The latter implies that for $n \geq 1, W_{n}$ can be decomposed as

$$
W_{n}=\left(J f_{n}, Q f_{n}\right)+\left(x_{n}, a_{n}\right)
$$

with

(i) $f_{n} \in \ell_{1}$ satisfying $\left\|f_{n}\right\|_{1} \leq 1+\varepsilon$;

(ii) $\left\|\left(x_{n}, a_{n}\right)\right\| \leq \delta(1+\varepsilon)$.

Since $\left(f_{n}\right)_{n \geq 1}$ is a bounded sequence in $\ell_{1}$, we may assume (by passing to a subsequence if necessary) that for every $n \geq 1$,

$$
f_{n}=f_{0}+g_{n}+h_{n}
$$

where

(a) $\lim _{n \rightarrow \infty}\left\|f_{n}\right\|_{1}$ exists;

(b) weak $^{*}-\lim _{n \rightarrow \infty} f_{n}=f_{0}$;

(c) $\left(g_{n}\right)_{n \geq 1}$ is a disjointly supported sequence in $\ell_{1}$;

(d) $\lim _{n \rightarrow \infty}\left\|h_{n}\right\|_{1}=0$.

We claim that

$$
\lim _{n \rightarrow \infty}\left\|f_{n}\right\|_{1} \leq 3 \delta(1+\varepsilon) .
$$

To see this claim, let $N \geq 1$; then

$$
\begin{aligned}
\frac{1}{N} \sum_{n=1}^{N}\left\|f_{n}\right\|_{1} & \leq\left\|f_{0}\right\|_{1}+\frac{1}{N} \sum_{n=1}^{N}\left\|g_{n}\right\|_{1}+\frac{1}{N} \sum_{n=1}^{N}\left\|h_{n}\right\|_{1} \\
& =\left\|f_{0}\right\|_{1}+\frac{1}{N}\left\|\sum_{n=1}^{N} g_{n}\right\|_{1}+\frac{1}{N} \sum_{n=1}^{N}\left\|h_{n}\right\|_{1} \\
& \leq 2\left\|f_{0}\right\|_{1}+\frac{1}{N}\left\|\sum_{n=1}^{N} f_{n}\right\|_{1}+\frac{2}{N} \sum_{n=1}^{N}\left\|h_{n}\right\|_{1} .
\end{aligned}
$$

Note that $\left\|f_{0}\right\|_{1} \leq \underline{\lim }_{N \rightarrow \infty} N^{-1}\left\|\sum_{n=1}^{N} f_{n}\right\|_{1}$ and $\lim _{N \rightarrow \infty} N^{-1} \sum_{n=1}^{N}\left\|h_{n}\right\|_{1}=0$. We deduce that

$$
\lim _{n \rightarrow \infty}\left\|f_{n}\right\|_{1} \leq 3 \varlimsup_{N \rightarrow \infty} \frac{1}{N}\left\|\sum_{n=1}^{N} f_{n}\right\|_{1} .
$$


We observe that

$$
\begin{aligned}
\frac{1}{N}\left\|\sum_{n=1}^{N} f_{n}\right\|_{1} & =\frac{1}{N}\left\|\sum_{n=1}^{N} J f_{n}\right\|_{\infty} \\
& \leq \frac{1}{N}\left\|\sum_{n=1}^{N}\left(J f_{n}, Q f_{n}\right)\right\| \\
& \leq \frac{1}{N}\left\|\sum_{n=1}^{N} W_{n}\right\|+\frac{1}{N}\left\|\sum_{n=1}^{N}\left(x_{n}, a_{n}\right)\right\| \\
& \leq \frac{1}{N}\left\|\sum_{n=1}^{N} W_{n}\right\|+\delta(1+\varepsilon) .
\end{aligned}
$$

Since $\left(W_{n}\right)$ is equivalent to the unit vector basis of $c_{0}$, we have

$$
\lim _{N \rightarrow \infty} N^{-1}\left\|\sum_{n=1}^{N} W_{n}\right\|=0 .
$$

Combining all the above estimates, we get inequality (2.1.4).

We now show that if $\Pi$ is the projection from $X$ onto the closed linear span of $\left(W_{n}\right)_{n \geq 1}$ defined by $\Pi=\sum_{n=1}^{\infty} W_{n}^{*} \otimes W_{n}$, then

$$
|||\Pi||| \geq \frac{1+\delta}{4 \delta(1+\varepsilon)^{2}}
$$

To see this, we first observe that for every $y^{*} \in \ell_{1}$,

$$
\left\|\left|\left(0, y^{*}\right)\right|\right\|=\left\|y^{*}\right\|_{1}(1+\delta) .
$$

For every $n \geq 1$, we have

$$
\begin{aligned}
1 & =\left\langle W_{n}^{*}, W_{n}\right\rangle \\
& =\left\langle\left(y_{n}^{*}, b_{n}^{*}\right),\left(J f_{n}+x_{n}, Q f_{n}+a_{n}\right)\right\rangle \\
& =\left\langle y_{n}^{*}, J f_{n}+x_{n}\right\rangle+\left\langle b_{n}^{*}, Q f_{n}+a_{n}\right\rangle \\
& \leq\left(\left\|y_{n}^{*}\right\|+\left\|b_{n}^{*}\right\|\right)\left(\left\|f_{n}\right\|_{1}+\delta(1+\varepsilon)\right) .
\end{aligned}
$$

Taking limits as $n \rightarrow \infty$, we deduce that

$$
\varliminf_{n \rightarrow \infty}\left\|b_{n}^{*}\right\|_{1} \geq \frac{1}{4 \delta(1+\varepsilon)}
$$

We can estimate ||$\Pi|| \mid$ as follows:

$$
\begin{aligned}
\| \Pi \Pi|| & \geq \sup _{n \geq 1} \frac{\left\|\left|W_{n}^{*}\right|\right\| \mid}{1+\varepsilon} \\
& =\sup _{n \geq 1} \frac{\left\|\left|\left(y_{n}^{*}, b_{n}^{*}\right)\right|\right\|}{1+\varepsilon} \\
& \geq \varliminf_{n \rightarrow \infty} \frac{\left\|\left|\left(y_{n}^{*}, b_{n}^{*}\right)\right|\right\| \mid}{1+\varepsilon} \\
& =\varliminf_{n \rightarrow \infty} \frac{||\left(0, b_{n}^{*}\right) \mid \|}{1+\varepsilon} .
\end{aligned}
$$

Thus (2.1.5) follows by combining (2.1.7) and (2.1.6). We conclude the proof by observing that ||$\Pi|\|\leq(1+\varepsilon)|||P|\|$. 
2.2. The $\ell_{1}$-case. Now we provide an example showing that Theorem 2 does not extend to the isometric case. In particular, the answer to Question 2 is negative.

First, recall that a norm $\|\cdot\|$ on a Banach space $E$ is said to be strictly convex if $\operatorname{Ext}\left(B_{E}\right)=S_{E}$. This is equivalent to the following property (see for instance 6. p. 246]):

$$
\text { If } x, y \in S_{E} \text { satisfy }\|x+y\|=2 \text {, then } x=y \text {. }
$$

It is clear from (2.2.1) that if $\|\cdot\|$ is strictly convex, then $(E,\|\cdot\|)$ does not contain any $\ell_{\infty}^{2}$ (the two dimensional $\ell_{\infty}$ ) isometrically. Indeed, if $e_{1}=(1,0)$ and $e_{2}=(0,1)$ are the unit vector basis of $\ell_{\infty}^{2}$, then $x=e_{1}$ and $y=e_{1}+e_{2}$ fail to satisfy (2.2.1).

Define a norm $|\cdot|$ on $\ell_{1}$ that is equivalent to the usual norm and such that its dual norm $|\cdot|^{*}$ is strictly convex. Such a dual norm on $\ell_{\infty}$ can be taken by setting:

$$
\left|\left(a_{i}\right)\right|^{*}:=\left(\left\|\left(a_{i}\right)\right\|_{\infty}^{2}+\sum_{i=1}^{\infty} 2^{-i}\left|a_{i}\right|^{2}\right)^{1 / 2} .
$$

Details on the existence of the norm $|\cdot|$ can be found in [6, pp. 241-254]. We define a Banach space $Y$ by setting:

$$
Y:=\left(C[0,1],\|\cdot\|_{\infty}\right) \oplus_{\infty}\left(\ell_{1},|\cdot|\right) .
$$

Proposition 4. The Banach space $Y$ contains a complemented subspace that is isometric to $\ell_{1}$ but $\ell_{1}$ is not isometric to a quotient of $Y$.

Let $\left(e_{n}\right)$ be the unit vector basis of $\ell_{1}$ and for $n \geq 1$, set $v_{n}:=e_{n} /\left|e_{n}\right|$. Fix a sequence $\left(f_{n}\right)_{n \geq 1}$ in $C[0,1]$ that is isometrically equivalent to the unit vector basis of $\ell_{1}$ and for $n \geq 1$, define $U_{n}:=\left(f_{n}, v_{n}\right) \in Y$. We claim that $\left(U_{n}\right)_{n \geq 1}$ is isometrically equivalent to the unit vector basis of $\ell_{1}$, and its closed linear span is complemented. In fact, for any finite sequence $\left(a_{n}\right)_{n \geq 1}$ of scalars,

$$
\begin{aligned}
\sum_{n \geq 1}\left|a_{i}\right| & =\left\|\sum_{n \geq 1} a_{n} f_{n}\right\|_{\infty} \\
& \leq\left\|\sum_{n \geq 1} a_{n} U_{n}\right\|_{Y} \\
& \leq \sum_{n \geq 1}\left|a_{i}\right|,
\end{aligned}
$$

therefore, $\sum_{n \geq 1}\left|a_{i}\right|=\left\|\sum_{n \geq 1} a_{n} U_{n}\right\|_{Y}$. Moreover, if we denote by $Z$ the closed linear span of $\left(U_{n}\right)_{n \geq 1}$, then $Z$ is a complemented subspace of $Y$. Indeed, let $T:\left(\ell_{1},|\cdot|\right) \rightarrow Y$ be defined by setting $T\left(v_{n}\right)=U_{n}$ for all $n \geq 1$ and $\Pi$ be the second projection from $Y$ onto $\left(\ell_{1},|\cdot|\right)$. Then $T \circ \Pi$ is a projection from $Y$ onto $Z$.

The fact that $\ell_{1}$ is not isometric to a quotient of $Y$ follows from the next lemma, which we assume is well known.

Lemma 5. Let $E$ and $F$ be Banach spaces and $T: c_{0} \rightarrow E \oplus_{1} F$ be an isometry. Then there exists $c_{j} \geq 0, j=1,2$ with:

(a) $c_{1}+c_{2}=1$;

(b) if $T=\left(T_{1}, T_{2}\right)$, then $\left\|T_{j}(e)\right\|=c_{j}\|e\|$ for $j=1,2$ and all $e \in c_{0}$.

In particular, if $E \oplus_{1} F$ contains an isometric copy of $c_{0}$, then either $E$ or $F$ contains an isometric copy of $c_{0}$. 
Proof. Denote by $c_{00}$ the space of finitely supported sequences of scalars and let $\left(e_{n}\right)_{n \geq 1}$ be the unit vector basis of $c_{0}$. Write $T=\left(T_{1}, T_{2}\right)$ with $T_{1}: c_{0} \rightarrow E$ and $T_{2}: c_{0} \rightarrow F$. We shall verify that for every $x \in c_{00}$ with $\|x\|=1$,

$$
\left\|T_{j}(x)\right\|=\left\|T_{j}\left(e_{1}\right)\right\| \text { for } j=1,2 .
$$

To see this, we will show first that if $x$ and $y$ are disjointly supported unit vectors, then

$$
\left\|T_{j}(x)\right\|=\left\|T_{j}(y)\right\| \text { for } j=1,2 .
$$

Write $2 x=(x-y)+(x+y)$. Then

$$
\begin{aligned}
2 & =\|T(2 x)\|=\left\|T_{1}(2 x)\right\|+\left\|T_{2}(2 x)\right\| \\
& \leq\left(\left\|T_{1}(x-y)\right\|+\left\|T_{1}(x+y)\right\|\right)+\left(\left\|T_{2}(x-y)\right\|+\left\|T_{2}(x+y)\right\|\right) \\
& =\|T(x-y)\|+\|T(x+y)\|=2 .
\end{aligned}
$$

For $j=1,2$, set $a_{j}=\left\|T_{j}(x-y)\right\|+\left\|T_{j}(x+y)\right\|-2\left\|T_{j}(x)\right\|$. Then $\left(a_{1}, a_{2}\right)$ is a positive element of $\ell_{1}^{2}$ whose norm is equal to zero so

$$
2\left\|T_{j}(x)\right\|=\left\|T_{j}(x-y)\right\|+\left\|T_{j}(x+y)\right\|, \quad j=1,2 .
$$

By reversing the role of $x$ and $y$, we get (2.2.2).

Now, let $x \in c_{00}$ with $\|x\|=1$. Choose, $n>1$ so that $e_{n}$ and $x$ are disjointly supported. From (2.2.2),

$$
\left\|T_{j}(x)\right\|=\left\|T_{j}\left(e_{n}\right)\right\|=\left\|T_{j}\left(e_{1}\right)\right\|, \quad j=1,2 .
$$

Setting $c_{j}=\left\|T_{j}\left(e_{1}\right)\right\|$ for $j=1,2$ proves the lemma.

End of the proof of Proposition 4. If $\ell_{1}$ is isometric to a quotient of $Y$, then the dual space $Y^{*}=C[0,1]^{*} \oplus_{1}\left(\ell_{\infty},|\cdot|^{*}\right)$ contains an isometric copy of $\ell_{\infty}=\ell_{1}^{*}$ and hence of $c_{0}$. But since $|\cdot|^{*}$ is strictly convex and $C[0,1]^{*}$ is a $L_{1}$-space, this is in contradiction with Lemma 5 and thus completes the proof.

\section{CONCLUding REMARKS}

The notion of asymptotically isometric copies of $\ell_{1}$ (respectively, $c_{0}$ ) is closely related to James's distortion theorems. We recall that a Banach space $E$ is said to contain an asymptotically isometric copy of $\ell_{1}$ (respectively, $c_{0}$ ) if there exist a null sequence $\left(\varepsilon_{n}\right)_{n \geq 1}$ in $(0,1)$ and a sequence $\left(x_{n}\right)_{n \geq 1}$ in $E$ such that for all finite sequence $\left(t_{n}\right)_{n}$ of scalars:

$$
\sum_{n}\left(1-\varepsilon_{n}\right)\left|t_{n}\right| \leq\left\|\sum_{n} t_{n} x_{n}\right\| \leq \sum_{n}\left|t_{n}\right|,
$$

respectively,

$$
\sup _{n}\left(1-\varepsilon_{n}\right)\left|t_{n}\right| \leq\left\|\sum_{n} t_{n} x_{n}\right\| \leq \sup _{n}\left|t_{n}\right| .
$$

The norm introduced in the definition of the Banach space $Y$ can be used to provide examples confirming the optimality of James's distortion theorems. We refer to 4 for earlier examples.

Proposition 6. (a) $\left(\ell_{1},|\cdot|\right)$ does not contain any subspace asymptotically isometric to $\ell_{1}$.

(b) $\left(\ell_{\infty},|\cdot|^{*}\right)$ does not contain any subspace asymptotically isometric to $c_{0}$. 
Proof. These statements follow from the norm $|\cdot|^{*}$ being strictly convex and from some known results. First, containing an asymptotically isometric copy of $c_{0}$ and containing an isometric copy of $c_{0}$ is equivalent in a dual space (3]). Second, according to 2], a Banach space contains an asymptotically isometric copy of $\ell_{1}$ if and only if its dual contains an isometric copy of $L_{1}[0,1]$. But since $\left(\ell_{\infty},|\cdot|^{*}\right)$ is strictly convex, it does not contain any isometric copy of $\ell_{1}$, and therefore it cannot contain any isometric copy of $L_{1}[0,1]$. Part (a) was already observed in 2] where an explicit formula for $|\cdot|$ was given (see [2, Corollary 12]).

The proof of Proposition 4 yields that the last part of the conclusion of Proposition 4 can be strengthened to " $c_{0}$ is not isometric to a subspace of $Y^{*}$ ". However, in [3] Dowling proved that $\ell_{1}$ is a quotient of $X$ if and only if $c_{0}$ embeds isometrically into $X^{*}$, so the more natural statement involving $\ell_{1}$ is only formally weaker.

\section{REFERENCES}

[1] J. Diestel, Sequences and series in Banach spaces, Graduate Text in Mathematics, 92, Springer-Verlag, New York, 1984. MR0737004 (85i:46020)

[2] S. Dilworth, M. Girardi, and J. Hagler, Dual Banach spaces which contain an isometric copy of $L_{1}$, Bull. Polish Acad. Sci. Math. 48 (2000), 1-12. MR1751149 (2001e:46016)

[3] P. N. Dowling, Isometric copies of $c_{0}$ and $l^{\infty}$ in duals of Banach spaces, J. Math. Anal. Appl. 244 (2000), no. 1, 223-227. MR 1746799 (2000m:46036)

[4] P. N. Dowling, W. B. Johnson, C. J. Lennard, and B. Turett, The optimality of James's distortion theorems, Proc. Amer. Math. Soc. 125 (1997), no. 1, 167-174. MR1346969 (97d:46010)

[5] P. N. Dowling, N. Randrianantoanina, and B. Turett, Remarks on James's distortion theorems. II, Bull. Austral. Math. Soc. 59 (1999), no. 3, 515-522. MR1698052 (2000c:46022)

[6] M. Fabian, P. Habala, P. Hájek, V. Montesinos Santalucía, J. Pelant, and V. Zizler, Functional analysis and infinite-dimensional geometry, CMS Books in Mathematics/Ouvrages de Mathématiques de la SMC, 8, Springer-Verlag, New York, 2001. MR1831176 (2002f:46001)

Department of Mathematics, Texas A\&M University, College Station, Texas 77843

E-mail address: johnson@math.tamu.edu

Department of Mathematics and Statistics, Miami University, Oxford, Ohio 45056

E-mail address: randrin@muohio.edu 